\title{
Design of Efficient Dynamic Digital Channelization Structure
}

\author{
Chao Zhang ${ }^{\text {a)}, ~ H o n g ~ M a, ~ Y i w e n ~ J i a o ~}$ \\ Space Engineering University of PLA, Beijing 101416, China. \\ a) zhangchao_1003@163.com.
}

\begin{abstract}
In order to solve the problem of inflexible parameter settings in traditional dynamic channelization structure, a weighted overlap-add (WOLA) filter bank is proposed to implement dynamic channelization structure. The filter bank is deduced and emulated through MATLAB. The simulation results show that the WOLA dynamic channelization structure enhances the flexibility of parameter setting and the reconstruction error is within $5 \times 10-3$. Comparing with the Discrete Fourier transform (DFT) polyphase filter structure, the system reconstruction error is reduced by an order of magnitude, it is verified the feasibility and good reconstruction characteristics of the WOLA dynamic channelization structure.
\end{abstract}

Key words: dynamic channelization structure, weighted overlap-add(WOLA), feasibility.

\section{INTRODUCTION}

With the development of communication technology, the different communication standards and modulations continue to emerge [1]. The receiver bandwidth of wideband receiver contains many unk-nown signals whose carrier frequency and bandwidth are random. It has brought great challenges to the reconnaissance broadband signal.

Channelization is a key technique for extracting independent signals within the receiving bandwidt-h. The traditional channelization is achieved by DFT polyphaser filter structure and Multi-channel DDC structure. But it only applies to the situation where signals are known within the receiver bandw-idth. Therefore, it is significance to study an efficient and dynamic channelization structure.

Abu-Al-Saud [2] proposed a wideband channelization method based on nonuniform subband deco-mposition in 2004. The subband technology was first introduced in channelization and had breakthro-ugh significance. Bing Li [3] improved this structure in 2007 . He proposed using energy detection to determine the position of the signal and then confirming the subband combining scheme. It was really realized the dynamic channelization of blind signals. However, the above-mentioned dynamic chann-elization structure are based on the DFT polyphase filter structure whose decimating factor and chan-nel number must be equal. In order to break this restriction, a new dynamic channelization structure is proposed. The WOLA (Weighted overlap-add) filter bank [4] is introduced in the new structure to realize dynamic channelization. It is an efficient structure that makes setting of channel parameters more flexible and has a better reconstitution performance.

\section{MATHEMATICAL MODEL OF DYNAMIC CHANNELIZATION}

The development of filter banks drives the channelization technology [5]. Figure 1 shows the structure diagram of dynamic channelization. 


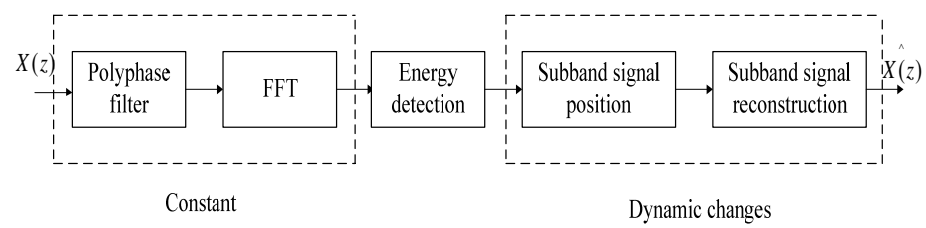

FIG.1. structure diagram of dynamic channelization

DFT Polyphase filter banks divide the input signals into several subbands. And synthesis filter ban-ks reconstruct the subbands containing the signal determined by energy detection. This structure dyn-amically determines reconstruction scheme based on energy detection. Therefore, it was realized the dynamic channelization of blind signals. But the DFT Polyphase filter banks limits the flexible of set-ting parameters. So, we need study a new flexible and efficient dynamical channelization structure.

\section{WOLA FILTER BANKS}

\section{WOLA Analysis Filter Bank}

In the DFT polyphase filter bank, the kth channel output signal can be expressed as [6]:

$$
\begin{aligned}
X_{k}(m) & =\left.\left[x(n) e^{-j w_{k} n}\right] * h(n)\right|_{n=m R} \\
& =\sum_{i=-\infty}^{\infty} h(m R-i) x(i) e^{-j w_{k} i}
\end{aligned}
$$

$\mathrm{R}$ is decimating factor. $\mathrm{K}$ is channel numbers. $w_{k}=2 \pi k / K$ expresses the center frequency of the kth channel. Assuming $q=i-m R$ :

$$
\begin{aligned}
X_{k}(m) & =\sum_{q=-\infty}^{\infty} h(-q) x(q+m R) e^{-j w_{k}(q+m R)} \\
& =e^{-j w_{k} m R} \sum_{q=-\infty}^{\infty} h(-q) x(q+m R) e^{-j w_{k} q} \\
& =e^{-j w_{k} m R} \tilde{X}_{k}(m) \\
\tilde{X}_{k}(m) & =\sum_{q=-\infty}^{\infty} h(-q) x(q+m R) e^{-j w_{k} q} \\
& =\sum_{q=-\infty}^{\infty} x_{m}(q) e^{-j w_{k} q}
\end{aligned}
$$

In equation (3), $x_{m}(q)=h(-q) x(q+m R)$. Since the number of channels is $\mathrm{K}$, the number of samples of output sequence is $\mathrm{K}$ in each $\mathrm{m}$ moment. Therefore $x_{m}(q)$ needs to be folded into a sequence of $\mathrm{K}$ points. Let $q=p+l K$ that $p=0,1, \cdots, K-1$, then: 


$$
\begin{aligned}
\tilde{X}_{k}(m) & =\sum_{p=0}^{K-1} \sum_{l=-\infty}^{\infty} x_{m}(p+l K) e^{-j w_{k}(p+l K)} \\
& =\sum_{p=0}^{K-1}\left[\sum_{l=-\infty}^{\infty} x_{m}(p+l K)\right] e^{-j w_{k} p} \\
& =\sum_{p=0}^{K-1} \tilde{x}_{m}(p) e^{-j w_{k} p} \\
\tilde{X}_{m}(p) & =\sum_{l=-\infty}^{\infty} x_{m}(p+l K) \\
& =\sum_{l=-\infty}^{\infty} x(m R+p+l K) h(-p-l K)
\end{aligned}
$$

According to the above formula, we can get the structure diagram of WOLA Analysis filter bank in figure 2 .

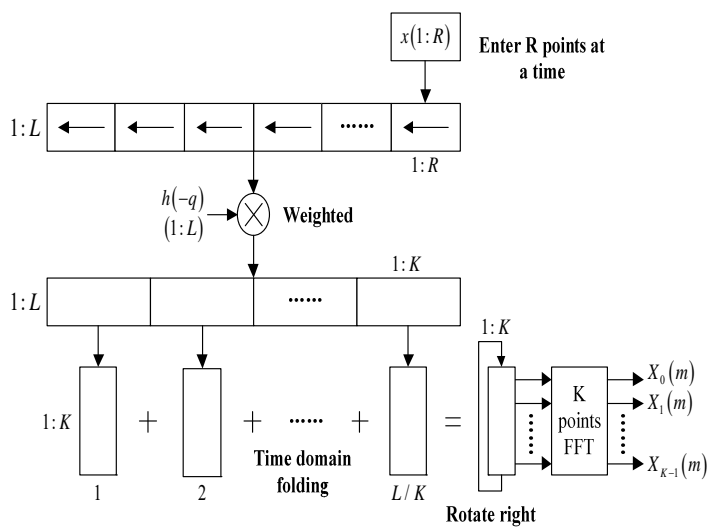

FIG.2. WOLA Analysis Filter Bank Implementation structure

The enter analysis filter bank signal processing flow can be divided into data segmentation, da-ta weighting, splicing accumulation, rotate right and FFT.

\section{WOLA Synthesis Filter Bank}

In the DFT polyphase filter bank, the synthe-sis of the output can be expressed as:

$$
\begin{aligned}
\hat{x(n)} & =\frac{1}{K} \sum_{k=0}^{K-1}\left[Y_{k}(n) * f(n) \times e^{j w_{k} n}\right] \\
& =\frac{1}{K} \sum_{k=0}^{K-1} \sum_{m=-\infty}^{\infty} X_{k}(m) f(n-m R) e^{j \omega_{k} n}
\end{aligned}
$$

Among (6), 


$$
Y_{k}(n)=\left\{\begin{array}{lr}
X_{k}(m) & n=m R \\
0 & \text { other }
\end{array}\right.
$$

Let $n=r+M R, \mathrm{r}=0,1 \ldots, \mathrm{R}-1$ and $\mathrm{M}$ is an integer.

$$
\begin{aligned}
\hat{x}(r+M R) & =\frac{1}{K} \sum_{k=0}^{K-1} \sum_{m=M-\alpha}^{M} X_{k} \hat{(m)} f(r+M R-m R) e^{j w_{k}(r+m R)} \\
& =\sum_{m=M-\alpha}^{M} f(r+(M-m) R) \frac{1}{K} \sum_{k=0}^{K-1} X_{k} \hat{(m)} e^{j w_{k} m R} e^{j w_{k}(r+(M-m) R)} \\
& =\sum_{m=M-\alpha}^{M} f(r+(M-m) R) \frac{1}{K} \sum_{k=0}^{K-1} \hat{\hat{\lambda}}(m) e^{j w_{k}(r+(M-m) R)}
\end{aligned}
$$

Among $(8), \hat{\hat{X}}_{k}(m)=\hat{X}_{k}(m) e^{j w_{k} m R \cdot \text { Let }}$

$$
\begin{aligned}
& U_{m}(r+(M-m) R) \\
& =\frac{1}{K} \sum_{k=0}^{K-1} \tilde{\hat{X}}_{k}(m) e^{j w_{k}(r+(M-m) R)}
\end{aligned}
$$

Because the $e^{j w_{k}(r+(M-m) R)}$ 's period is $\mathrm{K}$, so $e^{j w_{k}(r+(M-m) R)}=e^{j w_{k}(r+(M-m) R)_{\bmod K}}$.

$$
\begin{aligned}
U_{m}(r+(M-m) R) & =\frac{1}{K} \sum_{k=0}^{K-1} \tilde{\hat{X}}_{k}(m) e^{j w_{k}(r+(M-m) R)_{\bmod K}} \\
& =U_{m}\left((r+(M-m) R)_{\bmod K}\right)
\end{aligned}
$$

Substituting equation (10) into (8),

$$
\begin{aligned}
& \hat{x}(r+M R) \\
& =\sum_{m=M-\alpha}^{M} f(r+(M-m) R) U_{m}\left((r+(M-m) R)_{\bmod K}\right) \\
& =f(r) U_{M}(r)+f(r+R) U_{M-1}\left((r+R)_{\bmod K}\right)+ \\
& f(r+2 R) U_{M-2}\left((r+2 R)_{\bmod K}\right)+ \\
& \cdots+ \\
& f(r+\alpha R) U_{M-\alpha}\left((r+\alpha R)_{\bmod K}\right)
\end{aligned}
$$

According to the above formula, we can get the structure diagram of WOLA Synthesis filter bank in figure 3.

The enter synthesis filter bank signal processing flow can be divided into IFFT, loop left, data weighting and splicing accumulation. 


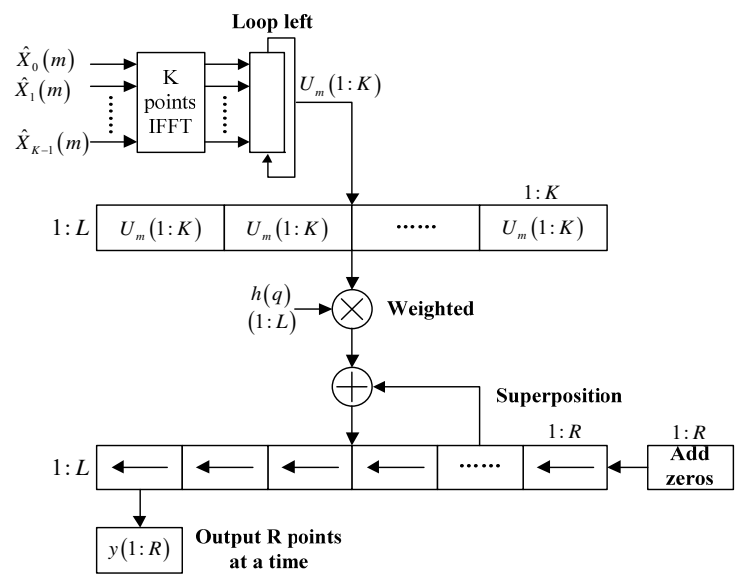

FIG.3. WOLA Synthesis Filter Bank Implementation structure

According to the above introduction, the advantages of WOLA structure can be drawn:

1 Releasing the multiples limitation of the number of channels $\mathrm{K}$ and the decimating factor of $\mathrm{R}$.

2 Weighting with filter coefficients instead of convolution greatly reduces the amout of system computation.

\section{Dynamic Digital Channelization Structure Based on WOLA Filter Bank}

Comprehensiving WOLA structure analysis filter bank and synthesis filter bank structure can get the dynamic digtial channelization structure based on WOLA filter bank in figure 4.

The input signal is divided into several subands by WOLA analysis filter bank. And then determining the position of the subchannel occupied by each subband signal by detection. Finally, the subbands which exist signal are inputed to synthesis filterbank to achieve signal's extraction.

This structure can make set of channel para-meters more flexible and has a better reconstitution performance.

\section{SIMULATION}

This section will verify the WOLA dynamic-al channelization structure. The sampling rate of the input signal is $\mathrm{fs}=1280 \mathrm{MSPS}$, the channel number is $\mathrm{K}=256$ and the decimating factor is $\mathrm{R}=176$. The sampling rate and the effective channel bandwidth of the channel baseband output are $f \mathrm{~s} / \mathrm{R}=7.3$ MSPS and $\mathrm{fs} / \mathrm{K}=5 \mathrm{MHz}$. A prototype filter with reconstructed characteristics is designed by raised cosine function. The length of prototype filter is 11264.The amplitude frequency characteristic curve of prototype filter is shown in figure 5. The sum of adjacent subband filters on power spectrum is shown in figure 6. 


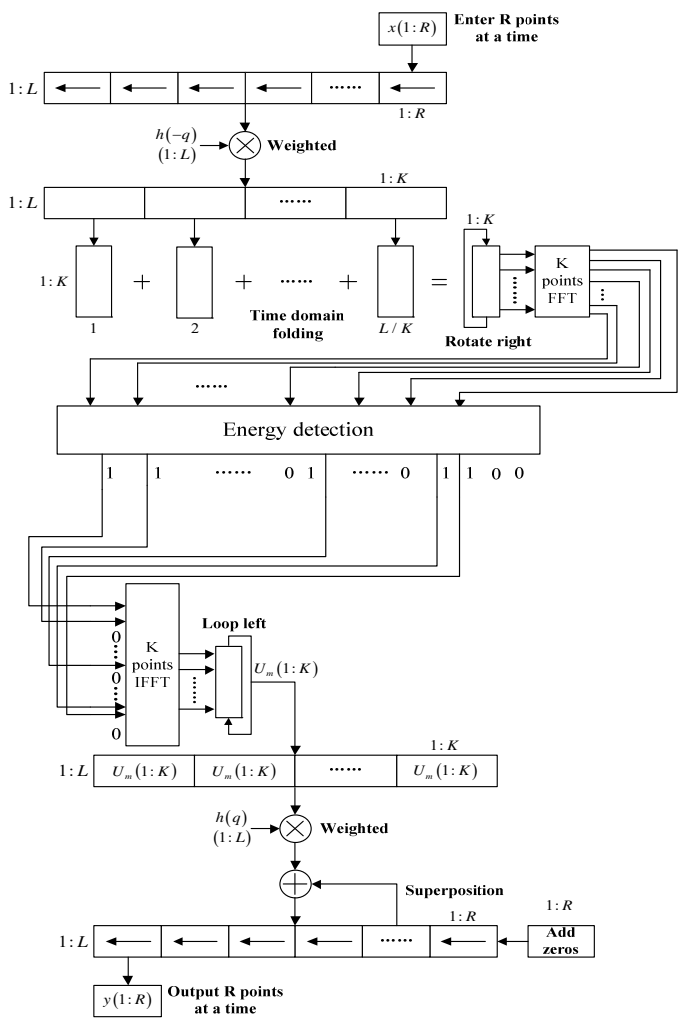

FIG.4. Dynamic Digtial Channelization Structure Based on WOLA Filter Bank

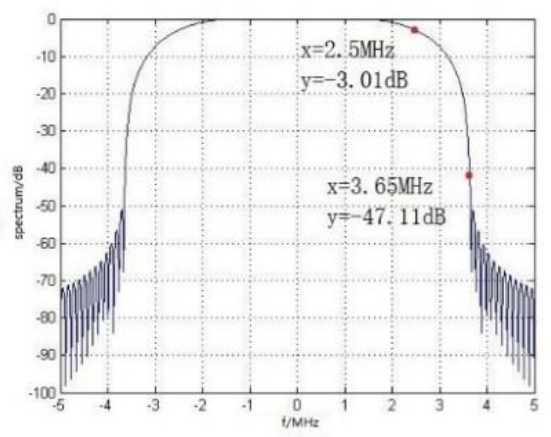

FIG.5. Amplitude-frequency response

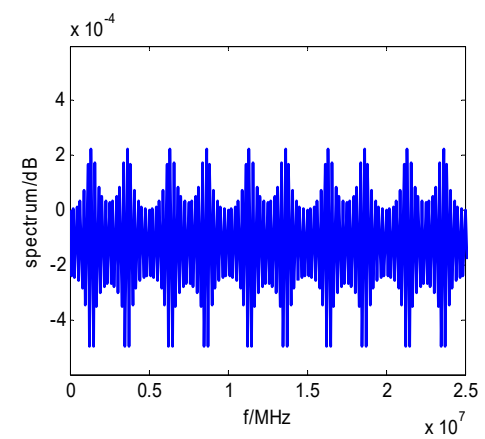

FIG.6. Subband power spectrum addition results 
The prototype filter bank, whose bandwidth is $5 \mathrm{MHz}$ and stopband frequency's attenuation is greater than $-40 \mathrm{db}$. Figure 6 shows the error of the sum of spectrum is within $1 \times 10-3 \mathrm{db}$. Therefore, this filter has good reconfiguration. Input test signal, one is sinusoidal signal, an-other is LFM signal.

$$
x(t)=\cos \left(2 \pi f_{0} t\right)+\cos \left(2 \pi f_{1} t+\pi \lambda t^{2}\right)
$$

$\mathrm{f} 0=99 \mathrm{MHz}, \mathrm{fl}=110 \mathrm{MHz}, \mathrm{B}=20 \mathrm{MHz}, \mathrm{T}=50 \mathrm{us}, \lambda=B / T$.According to system parameter settings, the sinusoidal signal should appear in 20th subband and LMF should appear in 22th to 26th subbands. T-he figure 7 shows the result of 19 th to 27 th subbands' specture.

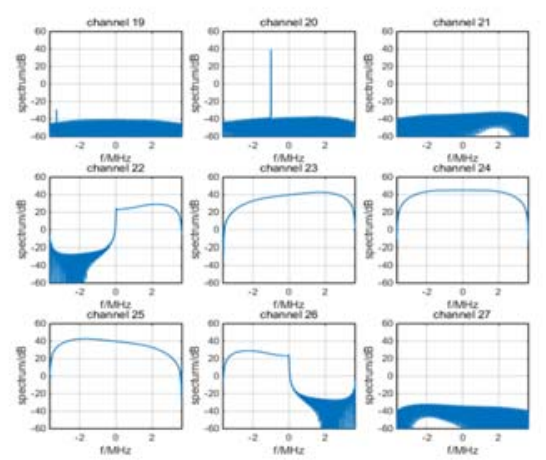

FIG.7. Subband spectrum

After energy detection, the subband existing signals are dynamically reconstruct. Figure 8 shows t-he spectrum of reconstruction signal. Figure 9 shows the comparison of reconstruction errors of DFT and WOLA structure. It can be seen from results that the WOLA dynamical channelization's reconstruction errors is less than $5 \times 10-3$ and the reconstruction errors of WOLA structure is much smaller than DFT structure. So, the WOLA dynamic channelization structure has a great reconstruction feature.

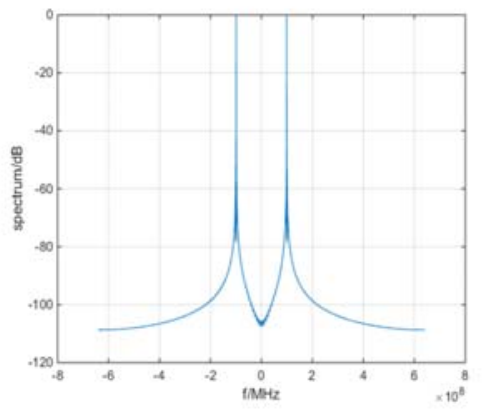

(a) Sinusoidal signal reconstruction spectrum

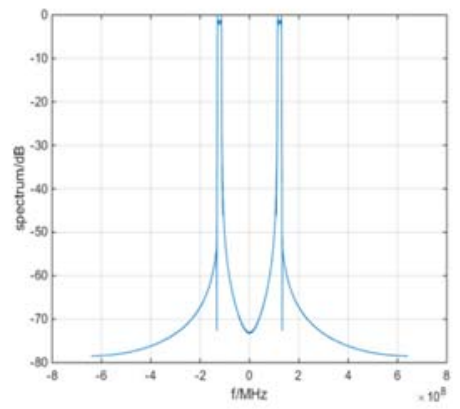

(b) LMF reconstruction spectrum

FIG.8. Spectrum of reconstruction signal 


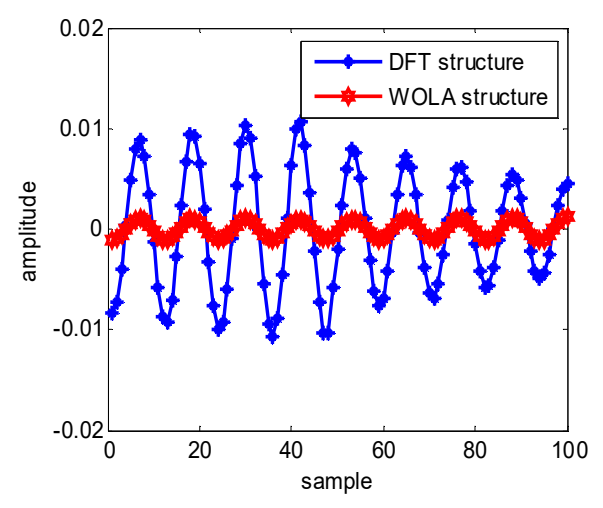

FIG.9. Comparison of reconstruction errors

\section{SUMMARY}

The dynamic channelization structure based on WOLA filter bank is proposed to improve t-he flexible of parameter setting. And the recon-struction errors is less than traditional structure.

It has important application value in the field of electronic reconnaissance.

\section{REFERENCES}

1. Zhao Yan. Subband technologies research and it's applications in wideband signal processing[D]. PLA Information Engineering University,2012.

2. Abu-Al-Saud W A and Studer G L. Efficient wideb-and channelizer for software radio systems using modulated PR filterbanks[J]. IEEE Transactions on Signal Processing 2004,52(10):2807-2820.

3. Ling Bing. Research on Channelization Techniques for Software Defined Radio[D]. PLA Information Engineering University,2007.

4. Wanghong, Lv Youxin, Wang Xuegang. Channeliz-ed Receiver with WOLA Filterbanks[J]. Journal of University of Electronic Science and Technology of China, 2008, 37(1):43-46.

5. Wang Guangyu. Multi-rate digital signal processing and filter bank theory [M]. Science Press,2013.

6. Wang Fang, Huang Zhen, Lu Jianhua. Efficient no-nuniform digital channelization and signal reconst-ruction techniques [J]. Telecommunications Techn-ology, 2011, 51(5):46-50. 